\title{
P116 ALCOHOL MISUSE IN THE UK ARMED FORCES
}

Terry Knight, Margaret Jones, Norman Jones, Mohammed Fertout, Neil Greenberg, Simon Wessely, Nicola T Fear King's College, London, UK

10.1136/oemed-2011-100382.330

Objectives There are concerns that alcohol misuse among military personnel could have serious consequences for both individual and organisational functioning. Research has found alcohol misuse to be particularly high in the UK Armed Forces, even when taking into account the UK general population's enthusiastic drinking behaviour. We aim to provide up-to-date information on alcohol misuse in the UK Armed Forces and explore changes in consumption.

Methods A two-phase cohort study of UK military personnel in which respondents completed questionnaires about military experiences and health outcomes. Data were collected between 2004 and 2006 and again between 2007 and 2009 (n=9984). Factors predicting new-onset alcohol misuse at phase 2 are explored using univariable and multivariable logistic regression. Results At phase two 13\% ( $n=1323)$ of personnel report alcohol misuse, which is associated with both deployment to Iraq and Afghanistan and with combat roles. Alcohol misuse has reduced among regular personnel who responded to both phases (from $14.2 \%$ to $10.9 \%$ ). Baseline factors associated with new-onset alcohol problems are being younger, being single, having a combat role, smoking, mental health difficulties, having parents with drink/drug problems. New-onset mental health difficulties and marital breakdown were also linked to new-onset alcohol problems, while military deployment and leaving the Armed Forces were not.

Conclusions Alcohol-reduction programmes may benefit from targeting resources at those most at risk (such as those in combat roles). Associations with other habits (ie, smoking) and personal difficulties (eg, divorce) indicate that combined welfare programmes may be beneficial in maintaining a healthy workforce. 